\title{
Recipient obesity as a risk factor in kidney transplantation
}

\author{
Uwe Scheuermann ${ }^{1 *}$, Jonas Babel ${ }^{1}$, Uta-Carolin Pietsch², Antje Weimann ${ }^{1}$, Orestis Lyros ${ }^{1}$, Katrin Semmling ${ }^{1}$, \\ Hans-Michael Hau ${ }^{1,3}$, Daniel Seehofer', Sebastian Rademacher ${ }^{1 \dagger}$ and Robert Sucher ${ }^{1+}$
}

\begin{abstract}
Background: The aim of the study was to investigate the effect of recipient obesity on the short- and long-term outcomes of patients undergoing primary kidney transplantation (KT).

Patients and methods: A total of 578 patients receiving primary KT in our department between 1993 and 2017 were included in the study. Patients were divided according to their body mass index (BMI) into normal weight (BMI $18.5-24.9 \mathrm{~kg} / \mathrm{m}^{2} ; \mathrm{N}=304$ ), overweight (BMI $25-29.9 \mathrm{~kg} / \mathrm{m}^{2} ; \mathrm{N}=205$ ) and obese (BMl $\geq 30 \mathrm{~kg} / \mathrm{m}^{2} ; \mathrm{N}=69$ ) groups. Their clinicopathological characteristics, outcomes, and survival rates were analyzed retrospectively.

Results: Obesity was associated with an increased rate of surgical complications such as wound infection $(P<0.001)$, fascial dehiscence $(P=0.023)$, and lymphoceles $(P=0.010)$. Furthermore, the hospital stay duration was significantly longer in the groups with obese patients compared to normal weight and overweight patients (normal weight: 22 days, overweight: 25 days, and obese: 33 days, respectively; $P<0.001)$. Multivariate analysis showed that recipient obesity (BMI $\geq 30$ ) was an independent prognostic factor for delayed graft function (DGF) (OR 2.400; 95\% Cl, $1.365-4.219 ; P=0.002)$ and postoperative surgical complications (OR 2.514; $95 \% \mathrm{Cl}, 1.230-5.136 ; P=0.011)$. The mean death-censored graft survival was significantly lower in obese patients (normal weight: $16.3 \pm 0.6$ years, overweight: $16.3 \pm 0.8$ years, obese $10.8 \pm 1.5$ years, respectively; $P=0.001$ ). However, when using the Cox proportional hazards model, the association between recipient obesity and death-censored renal graft failure disappeared, after adjustment for important covariates, whereas the principal independent predictors of graft loss were recipient diabetes mellitus and hypertension and kidneys from donors with expanded donor criteria.
\end{abstract}

Conclusion: In conclusion, obesity increases the risk of DGF and post-operative surgical complications after primary KT. Appropriate risk-adapted information concerning this must be provided to such patients before KT. Furthermore, obesity-typical concomitant diseases seem to negatively influence graft survival and need to be considered after the transplantation of obese patients.

Keywords: Kidney transplantation, Obesity, Body mass index, Outcome, Survival

*Correspondence: uwe.scheuermann@medizin.uni-leipzig.de

†Sebastian Rademacher and Robert Sucher contributed equally to this work and share senior authorship.

${ }^{1}$ Department of Visceral, Transplantation, Vascular and Thoracic Surgery, University Hospital of Leipzig, Liebigstrasse 20,04103 Leipzig, Germany Full list of author information is available at the end of the article

\begin{abstract}
Introduction
Kidney transplantation (KT) is the treatment of choice in patients with end-stage renal disease (ESRD) and it improves both patient survival and recipients quality of life compared to chronic dialysis treatment [1-3].

Due to an aging society and changes in lifestyle characterized by excessive calorie intake and a lack of physical activity - the percentage of overweight and
\end{abstract}


obese patients has steadily increased in recent decades. According to the current Organisation for Economic Co-operation and Development (OECD) data, 60\% of the population in Germany aged 15 years and older are overweight or obese [4]. Additionally, as obesity itself promotes ESRD, the proportion of obese renal transplant candidates is consequently increasing [5-9].

In general, surgery in obese patients is associated with a prolonged operative time and a higher risk for complications such as increased intraoperative blood loss and wound infections [10-13]. And higher prevalence of comorbidities in obese patients-such as cardiovascular disease, diabetes mellitus and hypertension - could jeopardize the allograft. Therefore, transplantation in obese recipients is still discussed controversially.

Thus, the current study sought to analyze the shortand long-term outcomes of obese ESRD patients undergoing primary KT.

\section{Patients and methods}

\section{Data collection and study population}

Medical data from all adult patients ( $\geq 18$ years of age) who underwent initial living or deceased donor kidney transplantation (KT) at the University Hospital of Leipzig between October 1993 and December 2017 were retrospectively analyzed. Our data source comprised a prospectively collected electronic database. Patients undergoing multi-organ (combined) transplants or re-transplants, underweight patients $(\mathrm{BMI}<18.5 \mathrm{~kg} /$ $\mathrm{m}^{2}$ ), and patients with missing (incomplete) data were excluded from the study. Follow-up data were collected until March 2020.

The characteristics of the study population included donor and recipient age, gender, and body mass index (BMI, weight in $\mathrm{kg} /$ height in $\mathrm{m}^{2}$ ), donor cause of death, duration of dialysis, time on the waiting list, and Criteria of Expanded Criteria Donors (ECD). Peri- and posttransplant data included information on the number of human leukocyte antigen (HLA) -A, B, and DR mismatches (0-6), last pretransplant panel reactive antibody (PRA) levels, cold (CIT) and warm ischemia time (WIT) of the grafts, duration of operation, as well as immunosuppressive therapy. CIT is defined as the time that the organ spent in cold preservation solution after removal from the donor, while WIT is the time from cross-clamping until cold perfusion, plus the time of implantation (organ out of ice until reperfusion).

\section{Outcome measures}

The outcome data included initial non-function (INF), biopsy-proven or clinically suspected episodes of acute rejection (in the first year after KT), delayed graft function (DGF), intra- and post-operative complications, date of graft failure, and patient death. INF was defined as dialysis dependence or creatinine clearance $\leq 20 \mathrm{~mL} / \mathrm{min}$ at three months post-transplant. Rejection episodes were histologically proved and DGF was defined as the need for dialysis in the first week following transplantation [14]. Post-operative complications occurring during the first three months after transplantation were analyzed. Complications included delayed wound healing, wound infection, urine leak, bleeding, development of hematoma, and lymphoceles. The Clavien-Dindo classification was used for morbidity assessment, and major morbidity was defined as being Clavien Dindo $3 \mathrm{~b}$ or greater [15]. New-onset diabetes after transplantation (NODAT) was defined as the need for insulin or oral hypoglycemic drugs. Graft failure was defined as a return to dialysis or re-transplantation. Post-operative deaths included all deaths occurring within 30 days after surgery.

\section{Body mass index}

Recipient body mass index (BMI) was calculated based on the formula: weight $(\mathrm{kg}) /$ [height $\left.(\mathrm{m})^{2}\right]$, from height and weight recorded at the time of transplantation. Patients were classified as normal weight (BMI 18.5$24.9 \mathrm{~kg} / \mathrm{m}^{2}$ ), overweight (BMI $25-29.9 \mathrm{~kg} / \mathrm{m}^{2}$ ), or obese $\left(\mathrm{BMI} \geq 30 \mathrm{~kg} / \mathrm{m}^{2}\right)$ according to guidelines of the World Health Organization (WHO) [16].

\section{Glomerular filtration rate}

Using serum creatinine levels, the estimated glomerular filtration rate (eGFR) was calculated using the Chronic Kidney Disease Epidemiology Collaboration (CKD-EPI) equation $\left(\mathrm{mL} / \mathrm{min} / 1.73 \mathrm{~m}^{2}\right.$ of standard body surface area (BSA)) [17]. To reduce errors induced by indexing the glomerular filtration rate for body surface area, the GFR was adjusted to the individual patient body surface area (eGFR $\mathrm{x}$ individual BSA $\left[\mathrm{m}^{2}\right] / 1,73 \mathrm{~m}^{2}$ standard $\mathrm{BSA}=\mathrm{mL} / \mathrm{min})[18,19]$.

\section{Standard and expanded criteria donors}

The standard criteria donor (SCD) was defined as a donor who is under 50 years of age and suffered brain death from any cause. Criteria of Expanded Criteria Donors (ECD) kidneys are sourced from donors over 60 years of age or donors between 50 and 59 years of age with at least two of the following three criteria: cerebrovascular death, arterial hypertension, and a donor serum creatinine level $>1.5 \mathrm{mg} / \mathrm{dl}[20]$.

\section{Organ procurement and transplantation}

The kidney grafts were procured according to the guidelines provided by Eurotransplant (ET) and transplanted into the iliacal fossa. Deceased donor kidneys were flushed in situ with cold HTK 
(histidine-tryptophan-ketoglutarate) solution and explanted. In living-related donation, kidneys were flushed with HTK after donor nephrectomy. For static cold storage, all grafts were immersed in HTK solution at $4{ }^{\circ} \mathrm{C}[21,22]$. The ureter was implanted into the bladder according to the Lich-Gregoir technique using a double J intra-ureteral splint $[23,24]$.

\section{Immunosuppression}

The initial immunosuppressive therapy comprised an induction therapy with the interleukin-2 receptor antagonists (daclizumab [withdrawn from the market in 2018] or basiliximab) or antithymocyte globulin, followed by triple maintenance immunosuppression comprising calcineurin inhibitors (tacrolimus or cyclosporine), and/ or mTOR inhibitors (everolimus or sirolimus), antimetabolites (azathioprine or mycophenolate mofetil), and tapered steroids (prednisolone). A rapid steroid-tapering regimen was applied in all our patients, starting with $500 \mathrm{mg}$ methylprednisolone intraoperatively to reach a dose of $25 \mathrm{mg}$ prednisolone at the end of the first week after transplantation. Further reduction intended a daily maintenance dose of $5 \mathrm{mg}$. Whenever possible, steroids were rapidly withdrawn and discontinued at the end of the first year post-transplant.

\section{Statistical analysis}

For comparison between the groups, the appropriate statistical significance test, including Student's t-test, the chi-squared test, analysis of variance (ANOVA), the Kruskal-Wallis test, and the Wilcoxon-Mann-Whitney test was used. Univariate and multivariate logistic regression analyses were used to evaluate the association between independent variables and binary outcomes of allograft function, and multivariate Cox proportional hazard analysis was applied to assess independent predictors of kidney graft failure. Prior to the regression analysis, post-operative complications were summarized into three groups: vascular (deep vein thrombosis, arterial or venous occlusion, secondary bleeding/hematoma), urological surgical (urine leakage), and surgical complications (wound infection, fascial dehiscence, lymphoceles). For the multivariate analyses, we used a forward stepwise regression model including only clinically relevant variables and those presenting $\mathrm{P}<0.05$ in univariate analysis. Survival rates were calculated using the Kaplan-Meier analysis, and the log-rank test was applied to test statistical significance. Graft survival was calculated as the time from initial transplant to graft failure (re-start of dialysis), uncensored for recipient death or censoring for death with a functioning graft. Patient survival was defined as the time from transplant to patient death, censoring for patients still alive at the time of analysis. If a recipient was alive or lost to follow-up at the time of the last contact, then survival time was censored at the time of the last contact. SPSS software, version 21.0 (SPSS Inc., Chicago, Illinois, USA) and Graphpad Prism software, version 9.2.0 (Graph-Pad Software Inc., La Jolla, CA) were used for statistical analysis and graphs. A P value $<0.05$ was considered as statistically significant. Baseline data are presented as median values with the standard deviation (SD).

\section{Results}

\section{Baseline characteristics}

Between October 1993 and December 2017, 947 kidney transplants (KT) were performed in our department. A total of 578 patients could be included in the analysis, with 304 normal weight (52.6\%), 205 overweight (35.5\%), and 69 obese (11.9\%) patients (Fig. 1).

The groups were similar in most of their baseline characteristics (Table 1). Median follow up was $5.8 \pm 6.8$ years. The proportion of obese patients significantly increased over the observation period (1993-2001: 7.0\%, 2002-2009: 12.9\%, and 2010-2017: 15.7\%, respectively; $P=0.011)$. A higher BMI was associated with a significantly longer duration of surgery $(P<0.001)$; however, no significant differences between the groups were found for cold and warm ischemia time or intraoperative complications.

\section{Outcome}

The analysis of post-operative outcome parameters is shown in Table 2 . In the overall study population, 32 kidneys lost their function in the first three months (initial non-function, INF) (normal weight, over-weight, and obese KT recipients: 11,16 , and 5, respectively, $P=0.108$ ), whereas permanent lack of graft function from the time of transplantation (primary non-function, PNF) was observed in five cases (normal weight, overweight, and obese KT recipients: 2, 3, and 0 , respectively, $P=0.447)$. No cases of PNF or INF were reported in kidney grafts after living donation.

Obese patients more frequently suffered from delayed graft function (DGF). These differences were most apparent in the subgroup of living donation (DGF normal weight, overweight, and obese, LD: 7 (11.3\%), 2 (7.7\%), and $3(60.0 \%)$, respectively, $P=0.005$; DD: $65(26.9 \%)$, $45(25.1 \%)$, and $29(45.3 \%)$, respectively, $P=0.005)$. Fig. 2 shows glomerular filtration (GFR) rates among the groups within the first six months after KT.

The length of time spent in the intensive care unit after KT was comparable between the three groups $(P=0.079)$. However, the number of patients receiving ventilation post-transplant was significantly higher in the obese group (normal weight, over-weight, and 


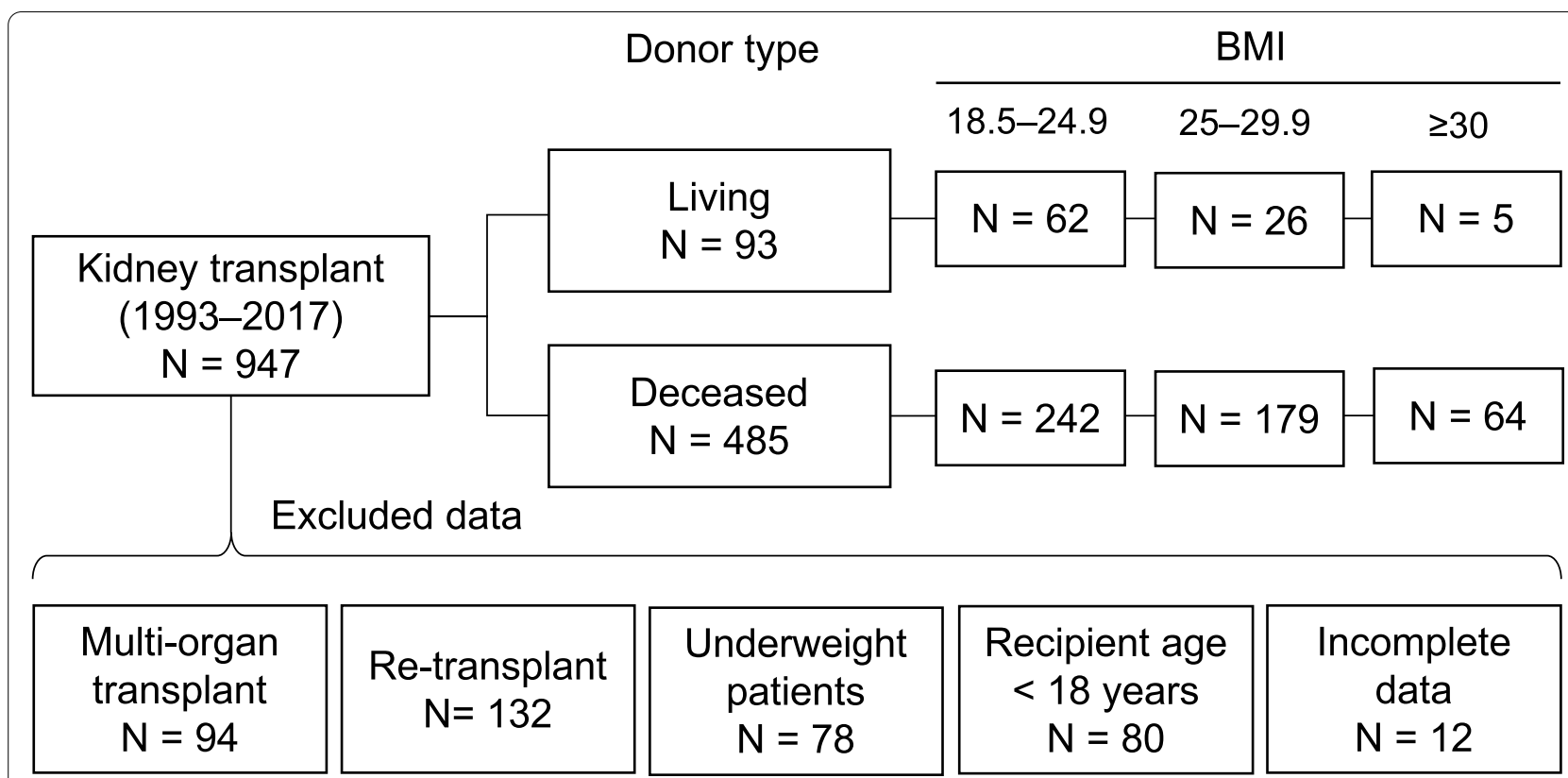

Fig. 1 Flow chart illustrating the inclusion and exclusion criteria of patients in the study. BMl, body mass index. Category numbers do not sum up due to overlap between exclusion criteria

obese KT recipients: $37(12.2 \%), 36(17.6 \%)$, and 16 (23.2\%), respectively, $P=0.041$ ), whereas the time of post-operative ventilation failed to show significance (normal weight, over-weight, and obese KT recipients: $2.0 \pm 6.2 \mathrm{~h}, 2.0 \pm 38.6 \mathrm{~h}$, and $2.75 \pm 33.1 \mathrm{~h}$, respectively, $P=0.347)$.

Comparing Clavien-Dindo scores $\geq 3 \mathrm{~b}$, there was a significant difference in major complication rate between the groups $(P=0.025)$. Obesity was especially associated with an increased rate of surgical complications such as wound infection $(P<0.001)$, fascial dehiscence $(P=0.023)$, and lymphoceles $(P=0.010)$. Univariate analysis revealed that especially a recipient $\mathrm{BMI} \geq 30$ is significantly correlated with postoperative surgical complications and graft dysfunction (Table 3). This was underlined by a significantly longer hospital stay of obese patients compared to normal weight and overweight patients (normal weight, over-weight, and obese KT recipients: 22 days, 25 days, and 33 days, respectively; $P<0.001$ ).

In the group of normal-weight and overweight patients, there were five in-hospital deaths each, whereas no in-hospital deaths were reported in the obese group $(\mathrm{P}=0.585)$. The causes of death included septic shock and multiple organ failure $(\mathrm{N}=3)$, liver insufficiency $(\mathrm{N}=1)$, and subdural hematoma $(\mathrm{N}=1)$ in the normal weight group and septic shock $(\mathrm{N}=1)$, endocarditis $(\mathrm{N}=1)$, acute bleeding with cardiac arrest $(\mathrm{N}=1)$ and acute heart failure $(\mathrm{N}=2)$ in the overweight group, respectively.
In the overall study period, acute rejection was clinically suspected or histologically proven in 184 (31.8\%) patients. In 149 cases, percutaneous kidney biopsies were performed and revealed acute rejection in 57 kidney allografts (38.3\%), without showing any significant difference between the three groups $(P=0.918)$. However, an increase in the total number of treated rejection episodes per KT could be demonstrated among the groups, although these trends were also not statistically significant (normal weight: 8.1/ 100 graft years, overweight: 9.6/ 100 graft years, and obese: 17.7/ 100 graft years, respectively; $P=0.133$ ) within the investigation period.

\section{Graft and patient survival}

Figure 3 shows Kaplan-Meier survival curves of grafts and patients after KT according to their BMI. The one-, three-, five- and ten-year cumulative death-censored graft survival rates were $92 \%, 88 \%, 83 \%$, and $72 \%$, respectively. The mean cumulative death-censored graft survival was $15.9 \pm 0.5$ years. Mean death-censored graft survival was significantly lower in obese patients (normal weight: $16.3 \pm 0.6$ years, overweight: $16.3 \pm 0.8$ years, obese $10.8 \pm 1.5$ years, respectively; $P=0.001$ ). After ten years of follow-up, graft survival was $39 \%$ in the obese group compared with $78 \%$ in the normal weight and $73 \%$ in the overweight group.

The one-, three-, five- and ten-year cumulative patient survival rates were $96 \%, 92 \%, 89 \%$, and $79 \%$, respectively. The mean patient survival was $18.7 \pm 0.5$ years. 
Table 1 Donor, recipient, and transplant characteristics

\begin{tabular}{|c|c|c|c|c|}
\hline \multirow[t]{2}{*}{ Variables } & \multicolumn{3}{|l|}{ Body mass index } & \multirow[t]{2}{*}{$P$-value } \\
\hline & $18.5-24.9(\mathrm{~N}=304)$ & $25-29.9(N=205)$ & $\geq 30(\mathrm{~N}=69)$ & \\
\hline \multicolumn{5}{|l|}{ Recipient } \\
\hline Age, years & $49.5 \pm 14.2$ & $55.9 \pm 11.9$ & $59.6 \pm 12.2$ & $<0.001$ \\
\hline Gender, male/ female (\%) & $182(59.9) / 122(40.1)$ & 135 (65.9)/ 70 (34.1) & $46(66.7) / 23(33.3)$ & 0.304 \\
\hline $\mathrm{BMI}, \mathrm{kg} / \mathrm{m}^{2}$ & $22.4 \pm 1.6$ & $27.1 \pm 1.3$ & $31.9 \pm 2.4$ & $<0.001$ \\
\hline $\begin{array}{l}\text { Time on the waiting list, } \\
\text { months }\end{array}$ & $17.7 \pm 28.6$ & $18.4 \pm 30.2$ & $23.0 \pm 30.3$ & 0.574 \\
\hline Dialysis duration, months & $47.3 \pm 35.5$ & $46.4 \pm 36.2$ & $55.6 \pm 33.6$ & 0.763 \\
\hline \multicolumn{5}{|l|}{ Cause of ESRD (\%) } \\
\hline Glomerulonephritis & $153(50.0)$ & $67(32.7)$ & $17(24.6)$ & $<0.001$ \\
\hline $\begin{array}{l}\text { Non-glomerulonephritis, cystic } \\
\text { kidney disease/ interstitial } \\
\text { nephritis/ diabetes mellitus/ } \\
\text { others/ unknown }\end{array}$ & $\begin{array}{l}47(15.5) / 28(9.2) / 3(1.0) / 50 \\
(16.4) / 24(7.9)\end{array}$ & $\begin{array}{l}39(19.0) / 26(12.7) / 16(7.8) / 42 \\
(20.4) / 15(7.3)\end{array}$ & $\begin{array}{l}15(21.7) / 7(10.1) / 10(14.6) / 16 \\
(23.2) / 4(5.8)\end{array}$ & \\
\hline \multicolumn{5}{|l|}{ Comorbidity (\%) } \\
\hline Diabetes mellitus & $26(8.6)$ & $30(14.6)$ & $22(31.9)$ & $<0.001$ \\
\hline Hypertension & $276(90.8)$ & $193(94.1)$ & $67(97.1)$ & 0.118 \\
\hline Coronary disease & $35(11.5)$ & $38(18.5)$ & $12(17.4)$ & 0.072 \\
\hline PVD & $11(3.6)$ & $16(7.8)$ & $6(8.7)$ & 0.071 \\
\hline \multicolumn{5}{|l|}{ Donor } \\
\hline Age, years & $51 \pm 15.6$ & $53 \pm 17.3$ & $56 \pm 16.4$ & 0.069 \\
\hline Gender, male/ female (\%) & $165(54.3) / 139(45.7)$ & $114(55.6) / 91(44.4)$ & $41(59.4) / 28(40.6)$ & 0.737 \\
\hline $\mathrm{BMI}, \mathrm{kg} / \mathrm{m}^{2}$ & $25.1 \pm 4.3$ & $25.0 \pm 3.7$ & $24.4 \pm 3.0$ & 0.200 \\
\hline $\begin{array}{l}\text { Organ quality, excellent/good/ } \\
\text { acceptable/ unknown (\%) }\end{array}$ & $\begin{array}{l}14(4.6) / 216(71.1) / 10(3.3) / 64 \\
(21.1)\end{array}$ & $\begin{array}{l}17(8.3) / 136(66.3) / 11(5.4) / 41 \\
(20.0)\end{array}$ & $5(7.2) / 50(72.5) / 4(5.8) / 10(14.5)$ & 0.329 \\
\hline \multicolumn{5}{|l|}{ Comorbidity (\%) } \\
\hline Diabetes mellitus & $22(7.2)$ & $13(6.3)$ & $5(7.2)$ & 0.921 \\
\hline Hypertension & $83(27.3)$ & $71(34.6)$ & $25(36.2)$ & 0.129 \\
\hline Donor type, LD/ SCD/ ECD (\%) & $62(20.4) / 146(48.0) / 96(31.6)$ & $26(12.7) / 100(48.8) / 79(38.5)$ & $5(7.2) / 32(46.4) / 32(46.4)$ & 0.014 \\
\hline \multicolumn{5}{|l|}{ Cause of death DD (\%) } \\
\hline CVA & $125(51.7)$ & $92(51.4)$ & $35(54.7)$ & 0.571 \\
\hline $\begin{array}{l}\text { Non-CVA, anoxia/ ischemia/ } \\
\text { polytrauma/ others }\end{array}$ & $\begin{array}{l}18(7.4) / 28(11.6) / 38(15.7) / 8 \\
(3.3)\end{array}$ & $\begin{array}{l}18(10.1) / 16(8.9) / 25(14.0) / 13 \\
(7.3)\end{array}$ & $7(10.9) / 4(6.3) / 9(14.1) / 3(4.7)$ & \\
\hline \multicolumn{5}{|l|}{ Transplant } \\
\hline \multicolumn{5}{|l|}{ Transplant era (\%) } \\
\hline $\begin{array}{l}1993-2001 / 2002-2009 / \\
2010-2017\end{array}$ & $108(35.5) / 105(34.5) / 91$ (29.9) & $64(31.2) / 71(34.6) / 70(34.1)$ & $13(18.8) / 26(37.7) / 30(43.5)$ & 0.079 \\
\hline HLA mismatches $\geq 3(\%)$ & $143(47.0)$ & $108(52.7)$ & $40(58.0)$ & 0.271 \\
\hline PRA (\%) & $50(16.4)$ & $29(14.1)$ & $10(14.5)$ & 0.753 \\
\hline Gender mismatch & $153(50.3)$ & $109(53.29$ & $31(44.9)$ & 0.462 \\
\hline CIT, hours & $11.5 \pm 7.1$ & $10.6 \pm 6.6$ & $11.4 \pm 5.7$ & 0.999 \\
\hline WIT, minutes & $40 \pm 19.9$ & $40 \pm 15.8$ & $45 \pm 23.8$ & 0.312 \\
\hline $\begin{array}{l}\text { Induction therapy, ATG/ } \\
\text { ILR2-RA }\end{array}$ & $15(4.9) / 101(33.2)$ & $7(3.4) / 68(33.2)$ & $2(2.9) / 26(37.7)$ & 0.582 \\
\hline \multicolumn{5}{|l|}{ Intra-operative complications (\%) } \\
\hline Bleeding & $11(3.6)$ & $10(4.9)$ & $3(4.3)$ & 0.780 \\
\hline Thrombosis artery & $8(2.6)$ & $6(2.9)$ & $3(4.3)$ & 0.748 \\
\hline Thrombosis vein & $4(1.3)$ & $2(1.0)$ & $1(1.4)$ & 0.925 \\
\hline Hyperacute rejection & $3(1.0)$ & 0 & 0 & 0.257 \\
\hline Duration of surgery, minutes & $172 \pm 46.0$ & $179 \pm 53.2$ & $194 \pm 64.8$ & $<0.001$ \\
\hline
\end{tabular}


Table 1 (continued)

\begin{tabular}{|c|c|c|c|c|}
\hline \multirow[t]{2}{*}{ Variables } & \multicolumn{3}{|l|}{ Body mass index } & \multirow[t]{2}{*}{$P$-value } \\
\hline & $18.5-24.9(\mathrm{~N}=304)$ & $25-29.9(N=205)$ & $\geq 30(N=69)$ & \\
\hline \multicolumn{5}{|l|}{ Immunosuppression (\%) } \\
\hline $\mathrm{CNI}, \mathrm{Tac} / \mathrm{CsA}$ & $170(55.9) / 128(42.1)$ & $122(59.5) / 75(36.6)$ & $47(68.1) / 22(31.9)$ & 0.400 \\
\hline mTOR inhibitor, Ever/ Siro & $1(0.3) / 8(2.6)$ & $0 / 4(2.0)$ & $1(1.4) / 1(1.4)$ & 0.471 \\
\hline $\mathrm{CNI}+$ mTOR inhibitor & $9(3.0)$ & $4(2.0)$ & $2(2.9)$ & 0.788 \\
\hline AM drug, AZA/ MMF & $23(7.6) / 262(86.2)$ & $8(3.9) / 180(87.8)$ & $3(4.3) / 63(91.3)$ & 0.502 \\
\hline Steroids, prednisolone & $295(96.7)$ & $191(93.2)$ & $69(100.0)$ & 0.134 \\
\hline Follow-up, years & $7.6 \pm 6.0$ & $6.7 \pm 5.9$ & $5.5 \pm 4.3$ & 0.005 \\
\hline
\end{tabular}

Data are shown as median \pm SD

AM Antimetabolite, Aza Azathioprin, ATG Anti-thymocyte globulin, BMI Body mass index, CNI Calcineurin inhibitor, CsA Ciclosporin A, CVA Cerebrovascular accident, CIT Cold ischemia time, DD Deceased donor, ECD Expanded criteria donor, ESRD End-stage renal disease, Ever Everolimus, HLA Human leukocyte antigen-A, B, and DR, IL2-RA Interleukin-2 receptor antagonist, LD Living donor, MMF Mycofenolate mofetil, $m T O R$ Mechanistic target of rapamycin, $P V D$ Peripheral vascular disease, $P R A$ Panel reactive antibody, SCD Standard criteria donor, Siro Sirolimus, WIT Warm ischemia time

The one-, three-, five- and ten-year survival rates, as well as the mean patient survival, were comparable between the groups (mean patient survival: normal weight: $19.2 \pm 0.6$ years, overweight: $17.6 \pm 0.7$ years, and obese: $15.8 \pm 1.5$ years, respectively; $P=0.186$ ).

The causes of graft loss are summarized in Table 4. The main reasons for graft failure were acute or chronic rejection (33.1\%) and chronic allograft nephropathy (22.3\%). Among the three BMI groups, the percentage of graft losses due to infection was significantly enhanced in obese KT recipients (6 (8.1\%), 5 (10.4\%), 9 (31.0\%); $P=0.006)$. No statistical difference could be observed between the three groups in regard to graft loss due to rejection (29 (39.2\%), 13 (27.1\%), 7 (24.1\%); $P=0.193)$.

In the multivariate regression analysis, obesity $(\mathrm{BMI} \geq 30)$ remained an independent predictor of DGF (OR 2.400; 95\% CI, 1.365-4.219; $P=0.002$ ) and postoperative surgical complications (OR 2.514; 95\% CI, 1.2305.136; $P=0.011$ ) (Table 5). However, after adjusting for important covariates, obesity failed to be an independent predictor of decreased graft survival or acute rejection. Independent predictors of graft loss were recipient diabetes mellitus and hypertension and kidneys from donors with expanded donor criteria. Expanded criteria donor was the only independent predictor of acute rejection (treated acute rejection (OR 1.448; 95\% CI, 1.131-1.852; $P=0.003)$, biopsy-proven acute rejection (OR 1.919; $95 \%$ CI, 1.236-2.978; $P=0.004)$ ).

\section{Discussion}

The proportion of obese patients receiving kidney transplantation (KT) has been increasing over the last decades [7-9] and is subsequently becoming one of the leading challenges for transplant surgeons. In the following, we will address the effect of obesity on the short- and longterm outcomes after living and deceased donor KT.

\section{Post-operative complications}

In the current study, obese KT recipients were significantly more likely to experience surgical complications such as wound infections, fascial dehiscence, and lymphoceles, compared with normal weight and overweight $\mathrm{KT}$ recipients. In reviewing the literature, wound complications were among the most common post-operative complications in obese patients after KT [25], and in accordance with the present results, previous publications have demonstrated that lymphoceles occur more often in obese patients after KT [26-28]. It is assumed that poor vascularization and the reduced immune response of fat tissue lead to poorer wound healing in this group of patients. Furthermore, a larger wound area and technical challenges due to more difficult exposure result in longer operation times and thus in higher probabilities of wound infection in obese patients [10-13]. The length of hospital stays, which is a surrogate marker for post-operative complications, was also significantly prolonged in the group of obese patients. This is in line with the retrospective study from Gore et al. published in 2006 about 20,000 patients after living or deceased KT, which revealed an independent effect of recipient BMI on the length of hospital stay in the adjusted analysis [29].

Despite a higher rate of major postoperative complications, no post-operative deaths occurred in the group with obese kidney grafts recipients within the first three months after KT in our study cohort. Especially cardiovascular risk factors must be excluded prior to $\mathrm{KT}$, as fatal cardiac events are a common cause of morbidity and death after KT in obese recipients [30-33]. Therefore, in our department, all patients routinely receive a cardiac 
Table 2 Post-operative outcome parameters and immunosuppression after deceased donor kidney transplantation

\begin{tabular}{|c|c|c|c|c|}
\hline \multirow[t]{2}{*}{ Variables } & \multicolumn{3}{|l|}{ Body mass index } & \multirow[t]{2}{*}{$P$-value } \\
\hline & $18.5-24.9(\mathrm{~N}=304)$ & $25-29.9(N=205)$ & $\geq 30(\mathrm{~N}=69)$ & \\
\hline \multicolumn{5}{|l|}{ Outcome Parameters } \\
\hline \multicolumn{5}{|l|}{ Surgical } \\
\hline RBC substitution (\%) & $83(27.3)$ & $55(26.8)$ & $17(24.6)$ & 0.903 \\
\hline FFP substitution (\%) & $25(8.2)$ & $15(7.3)$ & $4(5.8)$ & 0.775 \\
\hline Time on ICU, days & $5 \pm 5.0$ & $5 \pm 6.0$ & $5 \pm 14.6$ & 0.079 \\
\hline \multicolumn{5}{|l|}{ Post-operative complications (\%) } \\
\hline Deep vein thrombosis & $3(1.0)$ & $4(2.0)$ & $2(2.9)$ & 0.435 \\
\hline \multicolumn{5}{|l|}{ Occlusion or thrombosis } \\
\hline Renal artery & $5(1.3)$ & $4(2.0)$ & $2(2.9)$ & 0.631 \\
\hline Renal vein & $8(2.6)$ & $3(1.5)$ & $1(1.4)$ & 0.615 \\
\hline Pulmonary embolism & $2(0.7)$ & $1(0.5)$ & 0 & 0.788 \\
\hline Secondary bleeding/ hematoma & $62(20.4)$ & $39(19.0)$ & $16(23.2)$ & 0.754 \\
\hline Wound infection & $48(15.8)$ & $45(22.0)$ & $30(43.5)$ & $<0.001$ \\
\hline Fascial dehiscence & $1(0.3)$ & $5(2.4)$ & $3(4.3)$ & 0.023 \\
\hline Urine leakage & $12(4.0)$ & $3(1.5)$ & $2(2.9)$ & 0.266 \\
\hline Lymphocele & $37(12.2)$ & $19(9.3)$ & $16(23.2)$ & 0.010 \\
\hline Clavien-Dindo $\geq 3 b(\%)$ & $67(22.0)$ & $51(24.9)$ & $26(37.7)$ & 0.025 \\
\hline \multicolumn{5}{|l|}{ Renal } \\
\hline INF (\%) & $11(3.6)$ & $16(7.8)$ & $5(7.2)$ & 0.108 \\
\hline DGF (\%) & $72(23.7)$ & $47(22.9)$ & $32(46.4)$ & $<0.001$ \\
\hline Acute rejection, (\%) & $95(31.3)$ & $66(32.2)$ & $23(33.3)$ & 0.718 \\
\hline \multicolumn{5}{|l|}{$\mathrm{GFR}(\mathrm{mL} / \mathrm{min})$} \\
\hline POD7 & $36.5 \pm 42.5$ & $45.5 \pm 40.6$ & $18.1 \pm 28.3$ & 0.002 \\
\hline POD14 & $67.6 \pm 46.8$ & $62.0 \pm 36.8$ & $56.3 \pm 35.6$ & 0.041 \\
\hline POM1 & $82.5 \pm 36.5$ & $76.7 \pm 37.9$ & $78.9 \pm 35.9$ & 0.160 \\
\hline POM6 & $85.4 \pm 32.3$ & $80.9 \pm 35.7$ & $93.6 \pm 33.9$ & 0.704 \\
\hline \multicolumn{5}{|l|}{ Metabolic } \\
\hline NODAT (\%) & $15(4.9)$ & $12(5.8)$ & $8(11.6)$ & 0.110 \\
\hline Hospitalisation, days & $22 \pm 16.1$ & $25 \pm 16.2$ & $33 \pm 21.5$ & $<0.001$ \\
\hline Post-operative mortality 30 days (\%) & $3(1.0)$ & $3(1.5)$ & 0 & 0.579 \\
\hline Post-operative mortality 90 days (\%) & $6(2.0)$ & $5(2.4)$ & 0 & 0.436 \\
\hline
\end{tabular}

Data are shown as median \pm SD

DGF delayed graft function, FFP fresh frozen plasma, GFR glomerular filtration rate, ICU intensive care unit, INF initial non-function, NODAT new-onset diabetes mellitus after transplantation, $P O D$ post-operative day, $P O M$ post-operative month, $R B C$ red blood cells

stress test and, if necessary, a cardiac catheterization before enlisting for KT.

\section{Delayed graft function}

Consistent with previous publications, we could identify recipient obesity as an independent risk factor of delayed graft function (DGF) in our cohort [25]. In a retrospective single-center study of 1,132 kidney transplant recipients, Weissenbacher et al. also showed that recipient BMI correlates with the incidence of DGF after deceased donor KT (recipient BMI: OR 1.087; 95\% CI, 1.043-1.132; $\mathrm{P}<0.0001$ ) [34]. Additionally, a previously published retrospective multi-center study by Foucher et al. including 3,071 non-obese $(\mathrm{BMI}<30)$ and 615 obese $(\mathrm{BMI} \geq 30)$ recipients of kidney transplants confirmed this observation (recipient BMI: OR 1.89; 95\% CI, 1.562.29; $\mathrm{P}<0.0001$ ) [33].

Although the pathogenesis of DGF has not yet been fully elucidated, it is thought to be a result of immunologically and ischemia-induced graft injury [14, 35]. In our study, neither the duration of surgery, nor the ischemia time of the graft reached statistical significance concerning DGF $[29,34,36]$. However, it should be noted that there is an additional risk of DGF in obese patients when receiving kidneys from cadaveric donation, which have an increased susceptibility to ischemic injury [29, 35-37]. 


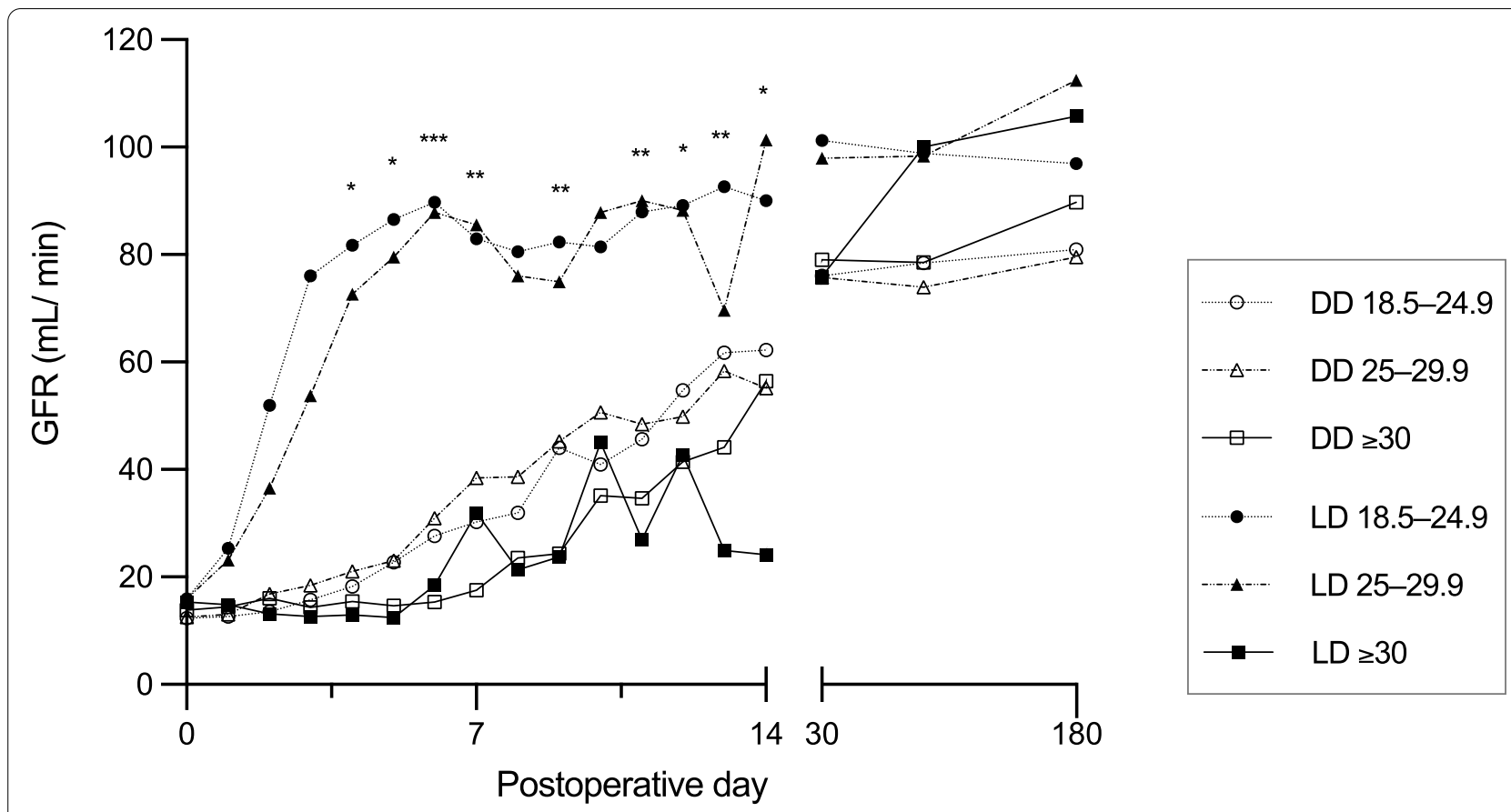

Fig. 2 Post-operative glomerular filtration rate according to recipient body mass index and donor type. BMl, body mass index; $D D$, deceased donor; GFR, glomerular filtration rate; LD, living donor. ${ }^{*} P<0.05,{ }^{* *} P<0.01,{ }^{* * *} P<0.001$

Table 3 Univariate analysis of recipient BMI and peri-operative kidney transplant outcome

\begin{tabular}{|c|c|c|c|c|c|c|c|}
\hline \multirow[t]{4}{*}{ Variables } & \multicolumn{7}{|c|}{ Body mass index } \\
\hline & \multirow[t]{3}{*}{$18.5-24.9$} & \multicolumn{3}{|c|}{$25-29.9$} & \multicolumn{3}{|l|}{$\geq 30$} \\
\hline & & \multicolumn{3}{|c|}{ Univariate analysis } & \multicolumn{3}{|c|}{ Univariate analysis } \\
\hline & & OR & $95 \% \mathrm{Cl}$ & P-value & OR & $95 \% \mathrm{Cl}$ & $P$-value \\
\hline \multicolumn{8}{|l|}{ Intraoperative complications } \\
\hline Bleeding (1) & ref & 1.366 & $0.569-3.278$ & 0.485 & 1.211 & $0.329-4.461$ & 0.774 \\
\hline Thrombosis artery or vein (1) & ref & 0.988 & $0.397-2.461$ & 0.980 & 1.106 & $0.304-4.030$ & 0.879 \\
\hline Duration of surgery (1) & ref & 1.391 & $0.960-2.016$ & 0.081 & 2.765 & $1.489-5.134$ & 0.001 \\
\hline Ventilation time (1) & ref & 1.524 & $0.871-2.665$ & 0.140 & 2.510 & $1.236-5.097$ & 0.011 \\
\hline Time on ICU (1) & ref & 0.756 & $0.528-1.082$ & 0.126 & 1.082 & $0.632-1.851$ & 0.773 \\
\hline \multicolumn{8}{|l|}{ Postoperative complications } \\
\hline Vascular (1) & ref & 0.897 & $0.586-1.374$ & 0.671 & 1.339 & $0.747-2.402$ & 0.327 \\
\hline Surgical (1) & ref & 1.131 & $0.783-1.633$ & 0.512 & 3.240 & $1.880-5.585$ & $<0.001$ \\
\hline Urological surgical (1) & ref & 0.361 & $0.101-1.297$ & 0.118 & 0.726 & $0.159-3.322$ & 0.680 \\
\hline Clavien-Dindo $\geq 3$ (\%) & ref & 1.171 & $0.772-1.777$ & 0.457 & 2.148 & $1.225-3.734$ & 0.008 \\
\hline Hospitalisation (1) & ref & 1.222 & $0.856-1.743$ & 0.269 & 2.456 & $1.400-4.307$ & 0.002 \\
\hline \multicolumn{8}{|l|}{ Renal outcome } \\
\hline INF (1) & ref & 2.232 & $1.014-4.914$ & 0.046 & 2.092 & $0.702-6.234$ & 0.185 \\
\hline DGF (1) & ref & 0.946 & $0.622-1.440$ & 0.796 & 2.827 & $1.640-4.875$ & $<0.001$ \\
\hline Acute rejection, all (2) & ref & 1.121 & $0.861-1.458$ & 0.397 & 1.494 & $0.861-2.171$ & 0.035 \\
\hline Graft failure (death-censored) (2) & ref & 1.113 & $0.767-1.617$ & 0.573 & 2.405 & $1.512-3.827$ & $<0.001$ \\
\hline NODAT (1) & ref & 1.198 & $0.549-2.615$ & 0.650 & 2.527 & $1.026-6.224$ & 0.044 \\
\hline
\end{tabular}

Prior to regression analysis, post-operative complications were summarized into three groups: vascular (deep vein thrombosis, arterial or venous occlusion, secondary bleeding/ hematoma), urological surgical (urine leakage), and surgical complications (wound infection, fascial dehiscence, lymphoceles).1, Univariate log regression; 2, univariate cox regression; 95\% Cl 95\% confidence interval, DGF Delayed graft function, ICU Intensive care unit, INF Initial non-function, NODAT new-onset diabetes mellitus after transplantation, $O R$ odds ratio, Ref Reference 

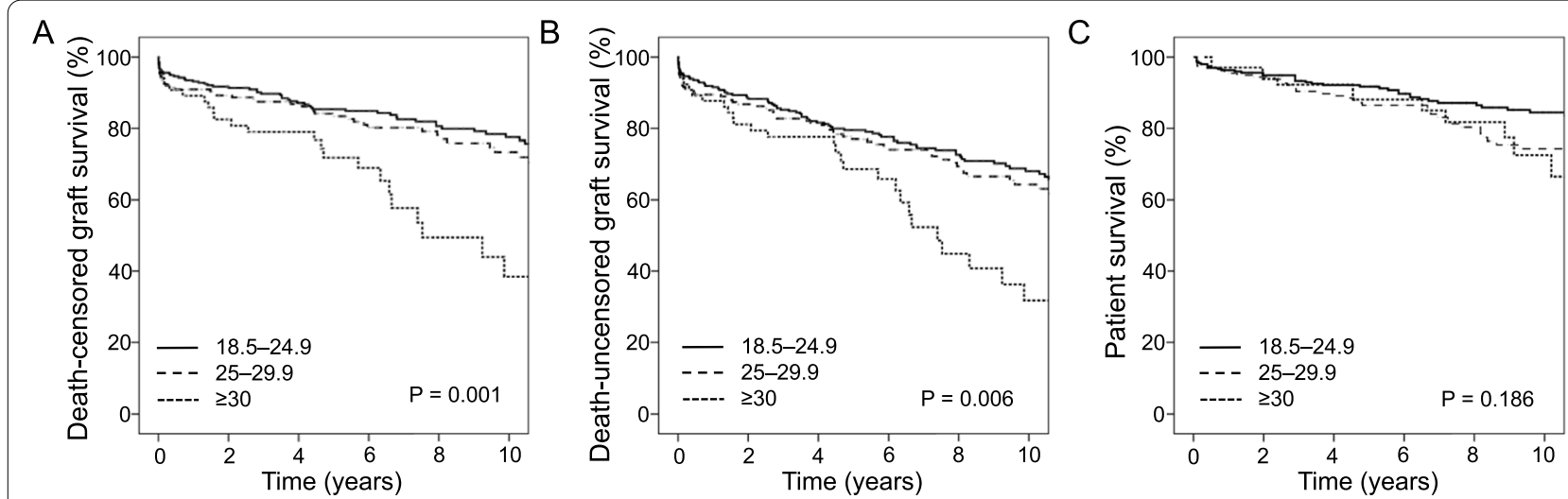

Fig. 3 Graft and patient survival according to the body mass index. A Death-censored kidney graft survival, B death-uncensored kidney graft survival and $\mathbf{C}$ patient survival ten years after kidney transplantation

Table 4 Causes of renal graft loss according to recipient body mass index. Category numbers do not sum up due to overlap between causes of graft failure (normal weight: $N=2$, obese: $N=1$ )

\begin{tabular}{|c|c|c|c|c|}
\hline \multirow[t]{2}{*}{ Variables } & \multicolumn{3}{|l|}{ Body mass index } & \multirow[t]{2}{*}{$P$-value } \\
\hline & $18.5-24.9(\mathrm{~N}=304)$ & $25-29.9(N=205)$ & $\geq 30(\mathrm{~N}=69)$ & \\
\hline \multicolumn{5}{|l|}{ Cause of graft loss (\%) } \\
\hline Death & $23(7.6)$ & $13(6.3)$ & $4(5.8)$ & 0.125 \\
\hline Graft failure & $72(23.7)$ & $48(23.4)$ & $28(40.6)$ & 0.007 \\
\hline \multicolumn{5}{|l|}{ Cause of graft failure (\%) } \\
\hline Vascular & $7(9.5)$ & $8(16.7)$ & $3(10.3)$ & 0.505 \\
\hline Acute or chronic rejection & $29(39.2)$ & $13(27.1)$ & $7(24.1)$ & 0.193 \\
\hline Recurrence of renal disease & $4(5.4)$ & $2(4.2)$ & $1(3.4)$ & 0.893 \\
\hline Chronic allograft nephropathy & $19(25.7)$ & $8(16.7)$ & $6(20.7)$ & 0.452 \\
\hline Infection & $6(8.1)$ & $5(10.4)$ & $9(31.0)$ & 0.006 \\
\hline Other & $9(12.2)$ & $12(25.0)$ & $3(10.3)$ & 0.130 \\
\hline
\end{tabular}

Therefore, the percentage of DGF after deceased donor $\mathrm{KT}$ is inherently higher compared to living donor $\mathrm{KT}$, as the prevalence of DGF ranges from 4 to $10 \%$ in patients after living KT, and between 2 and $50 \%$ in kidneys from brain-dead donors $[35,38]$. In our study, the rate of DGF was remarkably high in the subgroup of obese patients receiving kidneys after living donation (60\%). However, due to the low number of patients in this group $(\mathrm{N}=5)$, a conclusive statement is not possible and further studies with larger patient number are needed.

Furthermore, it should be noted that in obese KT recipients, renal function was only reduced in the early postoperative period after transplantation. Six months after KT, eGFR were comparable between the three study groups, indicating a good graft function even in KT recipients with a higher BMI.

\section{Acute rejection episodes}

A tendency towards a higher frequency of acute rejections per graft could be observed in the obese group of our patients. Meta-analysis showed that patients with a higher BMI have a significantly greater risk of acute rejection after KT [26]. Especially morbid obesity $(B M I \geq 35)$ demonstrated an association with acute rejection [29, 39]. A more difficult dosage adjustment, the maintenance of an appropriate level of immunosuppression, and a "state of chronic low-grade inflammation " in obese patients could be reasons for the enhanced rate of acute rejection in this group [40-42]. In our analysis, due to low patient numbers ( $\mathrm{BMI} \geq 35, \mathrm{~N}=8$ ), we could not evaluate the effects of an extreme BMI on outcomes after kidney transplantation. Further studies are necessary to analyze the serum levels of immunosuppressants in obese KT recipients and, in conjunction with those from acute graft rejection. 
Table 5 Multivariate regression analysis of predictors of delayed graft function, surgical post-operative complications, rejection, and kidney graft loss after kidney transplantation

\begin{tabular}{|c|c|c|c|c|c|c|c|c|}
\hline \multirow[t]{3}{*}{ Variables } & \multicolumn{4}{|c|}{ Delayed graft function (1) } & \multicolumn{4}{|c|}{ Surgical complications (1) } \\
\hline & UVA & Multivariate analysis & & & UVA & Multivariate analysis & & \\
\hline & $P$-value & OR & $95 \% \mathrm{Cl}$ & $P$-value & $P$-value & $H R$ & $95 \% \mathrm{Cl}$ & $P$-value \\
\hline \multicolumn{9}{|l|}{ Recipient } \\
\hline Age & 0.001 & 1.535 & $1.003-2.350$ & 0.048 & 0.040 & NS & NS & NS \\
\hline \multicolumn{9}{|l|}{ BMI } \\
\hline $18.5-24.9$ vs. $25-29.9$ & 0.796 & 0.821 & $0.530-1.273$ & 0.379 & 0.512 & 1.061 & $0.631-1.784$ & 0.823 \\
\hline $18.5-24.9$ vs. $\geq 30$ & $<0.001$ & 2.400 & $1.365-4.219$ & 0.002 & $<0.001$ & 2.441 & $1.182-5.038$ & 0.016 \\
\hline Diabetes mellitus & 0.179 & & & & 0.075 & & & \\
\hline Hypertension & 0.512 & & & & 0.388 & & & \\
\hline Coronary disease & 0.043 & NS & NS & NS & 0.202 & & & \\
\hline \multicolumn{9}{|l|}{ Donor } \\
\hline Age & 0.077 & & & & 0.296 & & & \\
\hline Donor type, LD/ DD & 0.002 & 2.118 & $1.091-4.112$ & 0.027 & 0.071 & & & \\
\hline Cause of death, CVA/ non-CVA & 0.960 & & & & 0.457 & & & \\
\hline Diabetes mellitus & 0.358 & & & & 0.700 & & & \\
\hline Hypertension & 0.013 & NS & NS & NS & 0.027 & NS & NS & NS \\
\hline ECD & 0.001 & 1.538 & $1.016-2.328$ & 0.042 & 0.194 & & & \\
\hline \multicolumn{9}{|l|}{ Transplant } \\
\hline WIT & 0.294 & & & & 0.003 & 1.906 & $1.183-3.071$ & 0.008 \\
\hline Duration of surgery & 0.017 & NS & NS & NS & 0.380 & & & \\
\hline Acute rejection & N/A & & & & 0.005 & 2.006 & $1.084-3.713$ & 0.027 \\
\hline \multicolumn{9}{|l|}{ B } \\
\hline \multirow[t]{3}{*}{ Variables } & \multicolumn{3}{|c|}{ Rejection (treated) (2) } & \multicolumn{5}{|c|}{ Graft failure (death-censored) (2) } \\
\hline & UVA & Multivariate analysis & & & UVA & Multivariate analysis & & \\
\hline & $P$-value & $H R$ & $95 \% \mathrm{Cl}$ & $P$-value & $P$-value & $H R$ & $95 \% \mathrm{Cl}$ & $P$-value \\
\hline \multicolumn{9}{|l|}{ Recipient } \\
\hline Age & 0.146 & & & & 0.051 & & & \\
\hline \multicolumn{9}{|l|}{ BMI } \\
\hline $18.5-24.9$ vs. $25-29.9$ & 0.397 & NS & NS & NS & 0.573 & NS & NS & NS \\
\hline $18.5-24.9$ vs. $\geq 30$ & 0.035 & NS & NS & NS & $<0.001$ & NS & NS & NS \\
\hline Diabetes mellitus & 0.034 & NS & NS & NS & 0.001 & 1.709 & $1.067-2.736$ & 0.026 \\
\hline Hypertension & 0.053 & & & & $<0.001$ & 2.565 & $1.514-4.344$ & 0.001 \\
\hline Coronary disease & 0.283 & & & & 0.194 & & & \\
\hline \multicolumn{9}{|l|}{ Donor } \\
\hline Age & 0.004 & NS & NS & NS & $<0.001$ & 2.725 & $1.841-4.033$ & $<0.001$ \\
\hline Donor type, LD/ DD & 0.046 & NS & NS & NS & 0.004 & NS & NS & NS \\
\hline Cause of death, CVA/ non-CVA & 0.191 & & & & 0.031 & NS & NS & NS \\
\hline Diabetes mellitus & 0.089 & & & & 0.011 & NS & NS & NS \\
\hline Hypertension & 0.182 & & & & $<0.001$ & NS & NS & NS \\
\hline ECD & 0.004 & 1.448 & $1.131-1.852$ & 0.003 & $<0.001$ & 3.095 & $2.115-4.530$ & $<0.001$ \\
\hline \multicolumn{9}{|l|}{ Transplant } \\
\hline WIT & 0.333 & & & & 0.497 & & & \\
\hline Duration of surgery & 0.144 & & & & 0.375 & & & \\
\hline Acute rejection & N/A & & & & $<0.001$ & 2.709 & $1.830-4.011$ & $<0.001$ \\
\hline
\end{tabular}

Following variables were tested in univariate analysis but failed to show significancy: cold ischemia time, delayed graft function; donor BMI, donor cause of death (CVA versus non-CVA), donor comorbidity diabetes mellitus, HLA, human leukocyte antigen-A, B, and DR (0-2 versus $\geq 3$ ); initial immunosuppression (calcineurin inhibitor versus mTOR inihibitor), induction therapy, initial non-function organ quality (excellent-good versus acceptable-poor), panel reactive antibodies, peripheral vascular disease; transplant era. 1, Univariate log regression; 2, univariate cox regression; 95\% CI 95\% Confidence interval, BMI Body mass index, CVA Cerebrovascular accident, DD Deceased donor, ECD Expanded criteria donor, HR Hazard ratio, LD Living donor, NS Not significant, OR Odds ratio, UVA Univariate analysis, WIT Warm ischemia time 


\section{Graft survival}

In our study, compared with normal weight or overweight KT recipients, graft survival in obese KT recipients was significantly reduced after five and ten years of observation. Several previous reports have shown that obesity itself confers a negative outcome on kidney graft survival [25, 31, 36, 39, 43-45]. However, after adjusting for important covariates, higher BMI was not an independent predictor for decreased graft survival in our analysis. Hence, the reasons for the increased risk of graft failure in overweight transplant recipients are most probably multifactorial. No correlation between the transplant era and patient outcome could be found in our data set. A higher prevalence of comorbidities in obese patients - such as cardiovascular disease, diabetes mellitus, and hypertension - could jeopardize the allograft, and an imbalance between donor and recipient weight might lead to renal hyperfiltration and graft injury [43]. In our study, obese patients were significantly older compared with non-obese patients at the time of $\mathrm{KT}$ and showed higher frequencies of secondary diseases of which the prevalence of diabetes mellitus and hypertension had an independent influence on all-cause graft failure. Especially since a new-onset diabetes mellitus is more often seen in obese patients after KT [33, 46, 47], this underlines the importance of strict follow-up care with intensive control of diabetes and high blood pressure to prevent or delay kidney graft failure.

\section{Limitations}

There are some limiting factors in this study. First, the retrospective non-randomized design of the study and single-center effect should be mentioned. Second, the long investigation period restricted data evaluation, thereby making further controlled and prospective studies necessary. Third, we solely used BMI to define obesity in our study, which may not be an appropriate measure to characterize the nutritional status of a patient. Therefore, further studies, with a measurement of body fat distribution and muscle mass, and their association with the risk of morbidity and mortality in transplant recipients would be of interest. Fourth, the influence of weight loss or gain on the KT outcome and possibilities of bariatric surgery could be highlighted in future studies.

\section{Conclusion}

In conclusion, data from our center indicate that obese patients after KT seem to suffer from surgical complications and DGF more frequently than non-obese patients. Therefore, appropriate risk-adapted information must be provided to these patient groups before KT. Furthermore, although obesity itself may not directly impair the survival of the kidney graft, secondary diseases such as diabetes mellitus and hypertension must be taken into consideration in obese KT recipients as they seem to negatively affect long-term graft survival.

\begin{abstract}
Abbreviations
BMI: Body mass index; CIT: Cold ischemia time; CNI: Calcineurin inhibitor; CVA: Cerebrovascular accident; DD: Deceased donor; DGF: Delayed graft function; ECD: Expanded criteria donors; ESRD: End-stage renal disease; Ever: Everolimus; FFP: Fresh frozen plasma; GFR: Glomerular filtration rate; INF: Initial non-function; KT: Kidney transplantation; LD: Living donor; MMF: Mycophenolate mofetil; NODAT: New-onset diabetes after transplantation; POD: Postoperative day; POM: Post-operative month; PVD: Peripheral vascular disease; SD: Standard deviation; Siro: Sirolimus; WIT: Warm ischemia time.
\end{abstract}

\section{Acknowledgements}

Not applicable

\section{Authors' contributions}

US and JB were responsible for the study conception and design; US, OL and JB were responsible for data acquisition; US, $\mathrm{HMH}, \mathrm{AW}$ and RS analyzed and interpreted the data; US, drafted the manuscript; and SR, KS, UCP, OL, DS and RS critically revised the manuscript. All authors read and approved the final manuscript.

\section{Funding}

Open Access funding enabled and organized by Projekt DEAL. This work was supported by the Open Access Publication Fund of the University of Leipzig. The funders had no role in study design, data collection and analysis, decision to publish, or preparation of the manuscript. The funders had no role in study design, data collection and analysis, decision to publish, or preparation of the manuscript. There are no potential conflicts of interest arising from associations with commercial or corporate interests in connection with the work submitted.

\section{Availability of data and materials}

Our database contains highly sensible data which may provide insight in clinical and personnel information about our patients and lead to identification of these patients. Therefore, according to organizational restrictions and regulations these data cannot be made publicly available. However, the datasets used and/or analyzed during the current study are available from the corresponding author on reasonable request.

\section{Declarations}

\section{Ethics approval and consent to participate}

The study was approved by the local ethical commission board from the University of Leipzig (AZ EK: 111-16-14032016). Written informed consent from any patient for data collection in a prospectively collected data base is available. However, written informed consent to the study was waived by the local Ethics Committee (Ethics Committee of the first affiliated University Hospital of Leipzig University) in view of the retrospective design of the study, accordingly the national and local guidelines such as the fact that all clinical/ laboratory measurements and procedures were part of the routine care.

\section{Competing interests}

The authors declare that they have no conflict of interest.

\section{Author details}

${ }^{1}$ Department of Visceral, Transplantation, Vascular and Thoracic Surgery, University Hospital of Leipzig, Liebigstrasse 20, 04103 Leipzig, Germany. ${ }^{2}$ Department of Anaesthesiology and Intensive Care Medicine, University Hospital of Leipzig, Leipzig, Germany. ${ }^{3}$ Department of Visceral, Thoracic and Vascular Surgery, University Hospital and Faculty of Medicine Carl Gustav Carus, Dresden University of Technology, Dresden, Germany. 
Received: 24 July 2021 Accepted: 11 January 2022

Published online: 18 January 2022

\section{References}

1. Rana A, Gruessner A, Agopian VG, Khalpey Z, Riaz IB, Kaplan B, Halazun KJ, Busuttil RW, Gruessner RW. Survival benefit of solid-organ transplant in the United States. JAMA Surg. 2015;150(3):252-9. https://doi.org/10.1001/ jamasurg.2014.2038. PMID: 25629390.

2. Sureshkumar KK, Patel BM, Markatos A, Nghiem DD, Marcus RJ. Quality of life after organ transplantation in type 1 diabetics with end-stage renal disease. Clin Transpl. 2006;20(1):19-25 PubMed PMID: 16556148.

3. Tonelli M, Wiebe N, Knoll G, Bello A, Browne S, Jadhav D, Klarenbach S, Gill J. Systematic review: kidney transplantation compared with dialysis in clinically relevant outcomes. Am J Transplant. 2011;11(10):2093-109. https://doi.org/10.1111/j.1600-6143.2011.03686.x. Epub 2011 Aug 30 PMID: 21883901.

4. OECD. Health at a Glance, 2019 OECD Indicators OECD Publishing, Paris 2021 https://doi.org/10.1787/4dd50c09-en;2019. Accessed 11 July 2021.

5. Tsujimoto T, Sairenchi T, Iso H, Irie F, Yamagishi K, Watanabe H, Tanaka K, Muto T, Ota H. The dose-response relationship between body mass index and the risk of incident stage $\geq 3$ chronic kidney disease in a general japanese population: the Ibaraki prefectural health study (IPHS). J Epidemiol. 2014;24(6):444-51. https://doi.org/10.2188/jea.je20140028 Epub 2014 Jul 5. PMID: 24998954; PMCID: PMC4213218.

6. Wang Y, Chen X, Song Y, Caballero B, Cheskin LJ. Association between obesity and kidney disease: a systematic review and meta-analysis. Kidney Int. 2008;73(1):19-33. https://doi.org/10.1038/sj.ki.5002586Epub 2007 Oct 10 PMID: 17928825

7. Hart A, Lentine KL, Smith JM, Miller JM, Skeans MA, Prentice M, Robinson A, Foutz J, Booker SE, Israni AK, Hirose R, Snyder JJ. OPTN/SRTR 2019 Annual Data Report: Kidney. Am J Transplant. 2021;21(Suppl 2):21-137. https://doi.org/10.1111/ajt.16502PMID: 33595191.

8. Kramer HJ, Saranathan A, Luke A, Durazo-Arvizu RA, Guichan C, Hou S, Cooper R. Increasing body mass index and obesity in the incident ESRD population. J Am Soc Nephrol. 2006;17(5):1453-9. https://doi.org/10. 1681/ASN.2005111241 Epub 2006 Apr 5 PMID:16597682.

9. McCullough KP, Morgenstern H, Saran R, Herman WH, Robinson BM. Projecting ESRD Incidence and Prevalence in the United States through 2030. J Am Soc Nephrol. 2019;30(1):127-35. https://doi.org/10.1681/ASN. 2018050531 Epub 2018 Dec 17. PMID: 30559143; PMCID: PMC6317596.

10. Ri M, Aikou S, Seto Y. Obesity as a surgical risk factor. Ann Gastroenterol Surg. 2017;2(1):13-21. https://doi.org/10.1002/ags3.12049 PMID:29863119; PMCID:PMC5881295.

11. Tjeertes EK, Hoeks SE, Beks SB, Valentijn TM, Hoofwijk AG, Stolker RJ. Obesity-a risk factor for postoperative complications in general surgery? BMC Anesthesiol. 2015:15:155. https://doi.org/10.1186/s12871-015-00967 Tjeertes,ElkeEKM[correctedtoTjeertes,EKM];Hoeks,SanneSE[correctedto Hoeks,SE];Beks,SabineSBJC[correctedtoBeks,SBJ];Valentign,TabitaTM[corre ctedtoValentijn,TM];Hoofwijk,AntonAGM[correctedtoHoofwijk,AGM];Sto. PMID:26228844;PMCID:PMC4520073.

12. Winfield RD, Reese S, Bochicchio K, Mazuski JE, Bochicchio GV. Obesity and the Risk for Surgical Site Infection in Abdominal Surgery. Am Surg. 2016:82(4):331-6 PMID: 27097626.

13. Doyle SL, Lysaght J, Reynolds JV. Obesity and post-operative complications in patients undergoing non-bariatric surgery. Obes Rev. 2010;11(12):875-86. https://doi.org/10.1111/j.1467-789X.2009.00700.x PMID: 20025695

14. Siedlecki A, Irish W, Brennan DC. Delayed graft function in the kidney transplant. Am J Transplant. 2011;11(11):2279-96. https://doi.org/10 1111/j.1600-6143.2011.03754.x Epub 2011 Sep 19. PMID: 21929642; PMCID: PMC3280444

15. Dindo D, Demartines N, Clavien PA. Classification of surgical complications: a new proposal with evaluation in a cohort of 6336 patients and results of a survey. Ann Surg. 2004;240:205-13.

16. World Health Organization. Obesity and overweight. https://www. who.int/news-room/fact-sheets/detail/obesity-and-overweight; 2021. Accessed 11 July 2021.

17. Levey AS, Stevens LA, Schmid CH, Zhang YL, Castro AF 3rd, Feldman HI, Kusek JW, Eggers P, Van Lente F, Greene T, Coresh J, CKD-EPI (Chronic
Kidney Disease Epidemiology Collaboration). A new equation to estimate glomerular filtration rate. Ann Intern Med. 2009;150(9):604-12. https://doi.org/10.7326/0003-4819-150-9-200905050-00006 Erratum in: Ann Intern Med. 2011 Sep 20;155(6):408. PMID: 19414839; PMCID: PMC2763564.

18. Du Bois D, Du Bois EF. A formula to estimate the approximate surface area if height and weight be known. 1916. Nutrition. 1989;5(5):303-11 discussion 312-3. PMID: 2520314

19. Zieschang S. "Genau richtig dosieren" bei Niereninsuffizienz - aber wie? Arzneiverordnung in der Praxis Band 47 Heft 1-2 März 2020 https://www akdae.de/Arzneimitteltherapie/AVP/Artikel/2020-1-2/018h/index.php; 2020. Accessed 20 July 2021.

20. Rao PS, Ojo A. The alphabet soup of kidney transplantation: SCD, DCD, ECD- fundamentals for the practicing nephrologist. Clin J Am Soc Nephrol. 2009;4(11):1827-31.

21. Eurotransplant. Chapter 9: The Donor. https://www.eurotransplant.org/ wp-content/uploads/2020/01/H9-The-Donor-Februar-2020.pdf; 2020 Accessed 11 July 2021.

22. Ladurner R, Steurer W. Technik der Multiorganentnahme. Viszeralchirurgie 2004;39:439-42.

23. Sollinger HW, Odorico JS, Becker YT, D'Alessandro AM, Pirsch JD. One thousand simultaneous pancreas-kidney transplants at a single center with 22-year follow-up. Ann Surg. 2009;250:618-30

24. Sollinger HW, Odorico JS, Knechtle SJ, D'Alessandro AM, Kalayoglu M, Pirsch JD. Experience with 500 simultaneous pancreas-kidney transplants. Ann Surg. 1998;228:284-96

25. Lafranca JA, IJermans JN, Betjes MG, Dor FJ. Body mass index and outcome in renal transplant recipients: a systematic review and metaanalysis. BMC Med. 2015;13:111. https://doi.org/10.1186/s12916-0150340-5 Erratum in: BMC Med. 2015;13:141. PMID: 25963131; PMCID: PMC4427990.

26. Furriel F, Parada B, Campos L, Moreira P, Castelo D, Dias V, Mota A. Pretransplantation overweight and obesity: does it really affect kidney transplantation outcomes? Transplant Proc. 2011;43(1):95-9. https://doi. org/10.1016/j.transproceed.2010.12.027 PMID: 21335163.

27. Orlic L, Mikolasevic I, Jakopcic I, Grskovic A, JelicPranjic I, Racki S, Stimac D. Body mass index: short- and long-term impact on kidney transplantation. Int J Clin Pract. 2015;69(11):1357-65. https://doi.org/10.1111/ijcp.12715 Epub 2015 Aug 12 PMID: 26268780.

28. Singh D, Lawen J, Alkhudair W. Does pretransplant obesity affect the outcome in kidney transplant recipients? Transplant Proc. 2005;37(2):717-20. https://doi.org/10.1016/j.transproceed.2004.12.033 PMID: 15848512.

29. Gore JL, Pham PT, Danovitch GM, Wilkinson AH, Rosenthal JT, Lipshutz GS, Singer JS. Obesity and outcome following renal transplantation. Am J Transplant. 2006;6(2):357-63. https://doi.org/10.1111/j.1600-6143.2005. 01198.x PMID: 16426321.

30. Grosso G, Corona D, Mistretta A, Zerbo D, Sinagra N, Giaquinta A, Caglià P, Amodeo C, Leonardi A, Gula R, Veroux P, Veroux M. The role of obesity in kidney transplantation outcome. Transplant Proc. 2012:44(7):1864-8. https://doi.org/10.1016/j.transproceed.2012.06.043 PMID: 22974857.

31. Aziz F, Ramadorai A, Parajuli S, Garg N, Mohamed M, Mandelbrot DA Foley DP, Garren M, Djamali A. Obesity: An Independent Predictor of Morbidity and Graft Loss after Kidney Transplantation. Am J Nephrol. 2020;51(8):615-23. https://doi.org/10.1159/000509105 Epub 2020 Jul 28 PMID: 32721967.

32. Lentine KL, Rocca-Rey LA, Bacchi G, Wasi N, Schmitz L, Salvalaggio PR, Abbott KC, Schnitzler MA, Neri L, Brennan DC. Obesity and cardiac risk after kidney transplantation: experience at one center and comprehensive literature review. Transplantation. 2008;86(2):303-12. https://doi.org/ 10.1097/TP.0b013e31817efOf9 PMID:18645495:PMCID:PMC2614624.

33. Foucher $Y$, Lorent M, Albano L, Roux S, Pernin V, Le Quintrec M, Legendre C, Buron F, Morelon E, Girerd S, Ladrière M, Glotz D, Lefaucher C, Kerleau C, Dantal J, Branchereau J, Giral M, DIVAT consortium. Renal transplantation outcomes in obese patients: a French cohort-based study. BMC Nephrol. 2021;22(1):79. https://doi.org/10.1186/s12882-021-02278-1 PMID: 33673808; PMCID: PMC7934368.

34. Weissenbacher A, Jara M, Ulmer H, Biebl M, Bösmüller C, Schneeberger S, Mayer G, Pratschke J, Öllinger R. Recipient and donor body mass index as important risk factors for delayed kidney graft function. Transplantation. 2012;93(5):524-9. https://doi.org/10.1097/TP.0b013e318243c6e4 PMID: 22362367. 
35. Perico N, Cattaneo D, Sayegh MH, Remuzzi G. Delayed graft function in kidney transplantation. Lancet. 2004;364(9447):1814-27. https://doi.org/ 10.1016/S0140-6736(04)17406-0 PMID: 15541456.

36. Cannon RM, Jones CM, Hughes MG, Eng M, Marvin MR. The impact of recipient obesity on outcomes after renal transplantation. Ann Surg. 2013;257(5):978-84. https://doi.org/10.1097/SLA.0b013e318275a6cb PMID: 23295317.

37. Molnar MZ, Kovesdy CP, Mucsi I, Bunnapradist S, Streja E, Krishnan M, Kalantar-Zadeh K. Higher recipient body mass index is associated with post-transplant delayed kidney graft function. Kidney Int. 2011;80(2):21824. https://doi.org/10.1038/ki.2011.114 Epub 2011 Apr 27. PMID: $21525853 ;$ PMCID: PMC5489124.

38. Yarlagadda SG, Coca SG, Formica RN Jr, Poggio ED, Parikh CR. Association between delayed graft function and allograft and patient survival: a systematic review and meta-analysis. Nephrol Dial Transplant. 2009;24(3):1039-47. https://doi.org/10.1093/ndt/gfn667 Epub 2008 Dec 22 PMID: 19103734.

39. Curran SP, Famure O, Li Y, Kim SJ. Increased recipient body mass index is associated with acute rejection and other adverse outcomes after kidney transplantation. Transplantation. 2014;97(1):64-70. https://doi.org/10. 1097/TP.0b013e3182a688a4 PMID: 24056619.

40. Shibata N, Hoshino N, Minouchi T, Yamaji A, Park K, Tomoyoshi T, Abe H, Kodama M. Relationship between area under the concentration versus time curve of cyclosporin A, creatinine clearance, hematocrit value, and other clinical factors in Japanese renal transplant patients. Int J Clin Pharmacol Ther. 1998;36(4):202-9 PMID: 9587046.

41. Okamoto Y, Christen T, Shimizu K, Asano K, Kihara S, Mitchell RN, Libby P. Adiponectin inhibits allograft rejection in murine cardiac transplantation. Transplantation. 2009;88(7):879-83. https://doi.org/10.1097/TP.0b013 e3181b6efbf PMID:19935458:PMCID:PMC2784664.

42. Quante M, Dietrich A, ElKhal A, Tullius SG. Obesity-related immune responses and their impact on surgical outcomes. Int J Obes (Lond). 2015;39(6):877-83. https://doi.org/10.1038/ijo.2015.21 Epub 2015 Feb 20 PMID: 25697667.

43. Bosma RJ, Kwakernaak AJ, van der Heide JJ, de Jong PE, Navis GJ. Body mass index and glomerular hyperfiltration in renal transplant recipients: cross-sectional analysis and long-term impact. Am J Transplant. 2007;7(3):645-52. https://doi.org/10.1111/j.1600-6143.2006.01672.x Epub 2007 Jan 22 PMID: 17250561.

44. Naik AS, Sakhuja A, Cibrik DM, Ojo AO, Samaniego-Picota MD, Lentine KL. The Impact of Obesity on Allograft Failure After Kidney Transplantation: A Competing Risks Analysis. Transplantation. 2016;100(9):1963-9. https:// doi.org/10.1097/TP.0000000000000983 PMID: 26569067.

45. Kostakis ID, Kassimatis T, Bianchi V, Paraskeva P, Flach C, Callaghan C, Phillips BL, Karydis N, Kessaris N, Calder F, Loukopoulos I. UK renal transplant outcomes in low and high BMI recipients: the need for a national policy. J Nephrol. 2020;33(2):371-81. https://doi.org/10.1007/s40620-019-00654-7 Epub 2019.

46. Gusukuma LW, Harada KM, Baptista AP, Alencar MR, de Sandes-Freitas TV, Tedesco-Silva H Jr, Medina-Pestana JO. Outcomes in obese kidney transplant recipients. Transplant Proc. 2014;46(10):3416-9. https://doi.org/10. 1016/j.transproceed.2014.09.112.PMID:25498063.Oct3 PMID: 31583535.

47. Moreira TR, Bassani T, de Souza G, Manfro RC, Gonçalves LF. Obesity in kidney transplant recipients: association with decline in glomerular filtration rate. Ren Fail. 2013;35(9):1199-203. https://doi.org/10.3109/08860 22X.2013.819735 Epub 2013 Aug 1 PMID: 23902319.

\section{Publisher's Note}

Springer Nature remains neutral with regard to jurisdictional claims in published maps and institutional affiliations.

Ready to submit your research? Choose BMC and benefit from:

- fast, convenient online submission

- thorough peer review by experienced researchers in your field

- rapid publication on acceptance

- support for research data, including large and complex data types

- gold Open Access which fosters wider collaboration and increased citations

- maximum visibility for your research: over 100M website views per year

At BMC, research is always in progress.

Learn more biomedcentral.com/submissions 